\title{
DELAY-DEPENDENT STABILITY AND STABILIZATION OF UNCERTAIN DISCRETE-TIME MARKOVIAN JUMP SINGULAR SYSTEMS WITH TIME DELAY
}

\author{
SHUPING MA ${ }^{12}$, XINZHI LIU $^{\bullet 34}$ and CHENGHUI ZHANG ${ }^{3}$
}

(Received 9 December, 2006; revised 5 June, 2007)

\begin{abstract}
This paper discusses robust stochastic stability and stabilization of time-delay discrete Markovian jump singular systems with parameter uncertainties. Based on the restricted system equivalent (RES) transformation, a delay-dependent linear matrix inequalities condition for time-delay discrete-time Markovian jump singular systems to be regular, causal and stochastically stable is established. With this condition, problems of robust stochastic stability and stabilization are solved, and delay-dependent linear matrix inequalities are obtained. A numerical example is also given to illustrate the effectiveness of this method.

2000 Mathematics subject classification: primary 39A12; secondary 93C55.

Keywords and phrases: discrete Markovian jump singular system, time-delay system, delaydependent, linear matrix inequality (LMI), robust stochastic stability and stabilization.
\end{abstract}

\section{Introduction}

In practice, many dynamical systems cannot be represented by linear time-invariant models since the dynamics of some features of these systems, such as for example, abrupt changes, breakdowns of components, changes in the interconnections of subsystems, etc., are random. Such classes of dynamical systems fall into the category of stochastic hybrid systems. A special class of hybrid systems referred to as Markovian jump systems, which exhibit random structures, has attracted much interest among researchers and many important problems involving these systems have been investigated, such as stability, stabilization and $H_{\infty}$ control problems, see $[4-6,8,18]$ and the

\footnotetext{
${ }^{1}$ School of Mathematics and System Science, Shandong University, Jinan, 250100, China; mashup@sdu.edu.cn.

${ }^{2}$ School of Computer Science and Technology, Shandong University, Jinan, China.

${ }^{3}$ School of Control and Engineering, Shandong University, Jinan, 250061, China.

${ }^{4}$ Department of Applied Mathematics, University of Waterloo, Waterloo, Ontario, Canada N2L 3G1;

xzliu@uwaterloo.ca.

(C) Australian Mathematical Society 2007, Serial-fee code 1446-1811/07
} 
references therein. On the other hand, time delay is commonly encountered in various engineering systems and is frequently a source of instability and poor performance. In general, the approaches to studing time-delay systems can be classified into two types: delay-dependent conditions, which include information on the length of delays $[3,4,7,8,10-13,19,20]$, and delay-independent conditions, which are applicable to delays of arbitrary length $[5,6,18]$. Since the stability of systems depends explicitly on their time delays, a delay-independent condition is often conservative, especially for small delays, while a delay-dependent condition is usually less conservative.

Singular systems, which are also referred to as implicit systems, or descriptor systems, have extensive applications in many practical systems, such as circuit boundary control systems, chemical processes, economy systems, and other areas, see [1,9]. So a great number of fundamental notions and results in control and system theory based on standard state-space systems have been extended successfully to singular systems $[1,2,9,14,15,17,21-24]$. In recent years, much attention has been focused on robust stability, robust stabilization and $H_{\infty}$ control problems for singular systems [2, 14, 15, 17, 21-24]. Xu and Lam [22] and Ma and Cheng [14] gave some results on robust stability and robust stabilization for discrete singular systems. The $H_{\infty}$ control problem for time-delay continuous-time singular systems was investigated in [17], [21] and [24],. For time-delay discrete singular systems, Xu, Lam and Yang [23] solved robust stabilization and $H_{\infty}$ control problems based on a delay-independent non-strict linear matrix inequality condition, $\mathrm{Ma}$ and Cheng [15] solved a robust stabilization problem based on a transformation of state-control pairs and a delaydependent LMI (linear matrix inequality). For Markovian jump singular systems, Boukas [2] discussed output feedback control for continuous-time Markovian jump singular systems. To the best of our knowledge, delay-dependent conditions for robust stochastic stability and robust stochastic stabilization problems for time-delay discrete Markovian jump singular systems have not been investigated in the literature.

The objective of this paper is to study robust stochastic stability and stabilization for time-delay discrete Markovian jump singular systems with parameter uncertainties. Based on the restricted system equivalent (RES) transformation and by introducing new state vectors, we shall transform the singular system into a time-delay discrete Markovian jump standard linear system, and then obtain some delay-dependent LMIs for the time-delay discrete Markovian jump singular systems to be regular, causal and stochastically stable. With this condition, we shall establish some criteria on robust stochastic stability and stabilization in terms of some delay-dependent LMIs. The rest of the paper is organized as follows. In Section 2, we shall formulate the problem and introduce some preliminaries. We then state and prove our main results in Section 3. Finally, we give, in Section 4, a numerical example to illustrate the effectiveness of the method, and some conclusive remarks in Section 5. 


\section{Description of problem and preliminaries}

Throughout this paper, for real symmetric matrices $X$ and $Y$, the notation $X \geq Y$ (respectively, $X>Y$ ) means that the matrix $X-Y$ is semipositive definite (respectively, positive definite). Here $I$ is the identity matrix with appropriate dimensions, the superscript " $T$ " represents the transpose, and $\operatorname{diag}[\cdots\}$ denotes a block-diagonal matrix. Also $\|x\|$ refers to the Euclidean norm of the vector $x$, that is, $\|x\|^{2}=x^{T} x$. Finally, $\mathscr{Z}$ denotes the set integers, $\mathbf{E}[\cdot]$ denotes the expectation and $*$ denotes the matrix entries implied by the symmetry of a matrix.

The system considered in this paper is assumed to be a state-space model as follows:

$$
\left\{\begin{aligned}
E x_{k+1} & =A\left(k, r_{k}\right) x_{k}+A_{d}\left(k, r_{k}\right) x_{k-d}+B\left(k, r_{k}\right) u_{k}, \quad k=-d, \ldots,-1,0, \\
x_{k} & =\phi(k),
\end{aligned}\right.
$$

where $k \in \mathscr{Z}, x_{k} \in R^{n}$ is the system state, $u_{k} \in R^{p}$ is the control input, $d$ is an unknown constant integer time delay, and $0<d \leq \bar{d}$, where $\bar{d}>0$ is a known integer and $\phi(k)$ is the initial value at $k$. Here $\left\{r_{k}, k \in \mathscr{Z}\right\}$ is a Markov chain taking values in finite space $\varphi=\{1,2, \cdots, N\}$, with transition probability from mode $i$ at time $k$ to mode $j$ at time $k+1$ given by

$$
p_{i j}=\operatorname{Pr}\left\{r_{k+1}=j \mid r_{k}=i\right\}
$$

with $p_{i j} \geq 0$ for $i, j \in \varphi$, and $\sum_{j=1}^{N} p_{i j}=1$. The matrix $E \in R^{n \times n}$ is singular, and $\operatorname{rank} E=r<n$. For each $i \in \varphi$, we have

$$
\begin{aligned}
& A(k, i)=A(i)+\delta A(k, i), \quad A_{d}(k, i)=A_{d}(i)+\delta A_{d}(k, i), \\
& B(k, i)=B(i)+\delta B(k, i)
\end{aligned}
$$

where $A(i), A_{d}(i)$ and $B(i)$ are known constant matrices with appropriate dimensions. Here $\delta A(k, i)$ and $\delta A_{d}(k, i), \delta B(k, i)$ are unknown matrices, denoting the uncertainties in System (2.1). In this paper, the uncertainties are assumed to be of the following form:

$$
\left[\delta A(k, i) \quad \delta A_{d}(k, i) \quad \delta B(k, i)\right]=E_{1}(i) \Delta(k, i)\left[\begin{array}{lll}
F_{1}(i) & F_{2}(i) & F_{3}(i)
\end{array}\right]
$$

where $E_{1}(i), F_{1}(i), F_{2}(i)$ and $F_{3}(i)$ are known constant matrices with appropriate dimensions and $\Delta(k, i) \in R^{q \times s}$ are unknown time-varying matrix functions satisfying

$$
\Delta^{T}(k, i) \Delta(k, i) \leq I .
$$

REMARK 1. The uncertainties $\delta A(k, i), \delta A_{d}(k, i)$ and $\delta B(k, i)$ are the so-called "norm-bounded uncertainties" and are considered frequently in robust control, see for example $[5,8,18]$ and the references therein. 
DEFINITION 2.1. Consider system (2.1) with $\delta A\left(k, r_{k}\right)=0, \delta A_{d}\left(k, r_{k}\right)=0$ and $u_{k}=0$.

(i) [9] For a given $r_{k}=i, i \in \varphi$, the pair $(E, A(i))$ is said to be regular if $\operatorname{det}(z E-A(i)) \not \equiv 0$.

(ii) [9] For a given $r_{k}=i, i \in \varphi$, the pair $(E, A(i))$ is said to be causal if it is regular and degree $(\operatorname{det}(z E-A(i)))=\operatorname{rank}(E)$.

(iii) System (2.1) with $\delta A\left(k, r_{k}\right)=0, \delta A_{d}\left(k, r_{k}\right)=0$ and $u_{k}=0$ is said to be regular and causal if every pair $(E, A(i))$ is regular and causal, for all $i \in \varphi$.

DEFINITION 2.2. (i) System (2.1) with $\delta A\left(k, r_{k}\right)=0, \delta A_{d}\left(k, r_{k}\right)=0$ and $u_{k}=0$ is said to be stochastically stable, if for every initial state $\left(\phi, r_{0}\right)$, the condition

$$
\mathbf{E}\left\{\sum_{k=0}^{\infty}\left\|x_{k}\left(\phi, r_{0}\right)\right\|^{2} \mid \phi, r_{0}\right\}<\infty
$$

is satisfied.

(ii) System (2.1) with $u_{k}=0$ is said to be robust stochastically stable if it is stochastically stable for all uncertainties satisfying (2.2) and (2.3).

(iii) System (2.1) is said to be robust stochastically stabilizable if there exists a state feedback controller

$$
u_{k}=K\left(r_{k}\right) x_{k}
$$

with $K(i)$, when $r_{k}=i$, a constant matrix such that the resulting closed-loop system is robust stochastically stable.

REMARK 2. (i) If System (2.1) with $\delta A\left(k, r_{k}\right)=0, \delta A_{d}\left(k, r_{k}\right)=0$ and $u_{k}=0$ is regular and causal, then for any initial value $\phi(k)$, there exists a unique solution of System (2.1) for each $r_{k}=i, i \in \varphi$.

In fact, since rank $E=r<n$, there exist two nonsingular matrices $M, N \in R^{n \times n}$ such that $M E N=\left[\begin{array}{ll}I_{r} & 0 \\ 0 & 0\end{array}\right]$ and we write

$$
M A(i) N=\left[\begin{array}{ll}
A_{1}(i) & A_{2}(i) \\
A_{3}(i) & A_{4}(i)
\end{array}\right], \quad M A_{d}(i) N=\left[\begin{array}{ll}
A_{d 1}(i) & A_{d 2}(i) \\
A_{d 3}(i) & A_{d 4}(i)
\end{array}\right] \text { and } x_{k}=N\left[\begin{array}{l}
x_{1 k} \\
x_{2 k}
\end{array}\right]
$$

Then System (2.1) with $\delta A\left(k, r_{k}\right)=0, \delta A_{d}\left(k, r_{k}\right)=0$ and $u_{k}=0$ is RSE to the following system:

$$
\left\{\begin{aligned}
x_{1(k+1)} & =A_{1}(i) x_{1 k}+A_{2}(i) x_{2 k}+A_{d 1}(i) x_{1(k-d)}+A_{d 2}(i) x_{2(k-d)}, \\
0 & =A_{3}(i) x_{1 k}+A_{4}(i) x_{2 k}+A_{d 3}(i) x_{1(k-d)}+A_{d 4}(i) x_{2(k-d)}, \quad k=-d, \ldots, 0 \\
{\left[\begin{array}{l}
x_{1 k} \\
x_{2 k}
\end{array}\right] } & =\left[\begin{array}{l}
\phi_{1}(k) \\
\phi_{2}(k)
\end{array}\right] .
\end{aligned}\right.
$$


Definition 2.1 and System (2.1) being regular and causal mean that the matrix $A_{4}(i)$ is nonsingular [9] for each mode $i \in \varphi$. Then it follows that

$$
\begin{aligned}
x_{1(k+1)}= & \left(A_{1}(i)-A_{2}(i) A_{4}^{-1}(i) A_{3}(i)\right) x_{1 k}+\left(A_{d 1}(i)-A_{2}(i) A_{4}^{-1}(i) A_{d 3}(i)\right) x_{1(k-d)} \\
& +\left(A_{d 2}(i)-A_{2}(i) A_{4}^{-1}(i) A_{d 4}(i)\right) x_{2(k-d)}, \\
x_{2 k}= & -A_{4}^{-1}(i)\left(A_{3}(i) x_{1 k}+A_{d 3}(i) x_{1(k-d)}+A_{d 4}(i) x_{2(k-d)}\right),
\end{aligned}
$$

which indicates that a unique solution of System (2.1) exists for each $i \in \varphi$.

(ii) By Definitions 2.1 and 2.2, one can see that regularity, causality and stochastic stability are preserved under an RES transformation.

(iii) When $E$ is nonsingular, System (2.1) can be transformed to a class of timedelay discrete Markovian jump standard linear systems. The problems of stochastic stability and stochastic stabilization for such systems have been solved in the literature $[4,6,18]$.

The purpose of this paper is to develop delay-dependent LMI conditions such that System (2.1) with $u_{k}=0$ is regular, causal and robust stochastically stable, and to design a state feedback controller of the form (2.4) such that the resulting closedloop system is regular, causal and robust stochastically stable for all uncertainties satisfying (2.2) and (2.3).

The following lemma will be used in the proof of the main results.

LEMMA 2.3 ([16]). Given a symmetric matrix $\Omega$ and matrices $\Gamma$ and $\Xi$ with appropriate dimensions, then

$$
\Omega+\Gamma \Delta \Xi+\Xi^{T} \Delta^{T} \Gamma^{T}<0
$$

for all $\Delta$ satisfying $\Delta^{T} \Delta \leq I$, if and only if there exists a scalar $\epsilon>0$ such that

$$
\Omega+\epsilon \Gamma \Gamma^{T}+\epsilon^{-1} \Xi^{T} \Xi<0 .
$$

\section{Main results}

In this section, first of all, we consider the regularity, causality and robust stochastic stability of System (2.1) with $u_{k}=0$. Since $\operatorname{rank} E=r<n$, there exist two nonsingular matrices $M$ and $N \in R^{n \times n}$ such that

$$
\left\{\begin{aligned}
M E N & =\left[\begin{array}{ll}
I_{r} & 0 \\
0 & 0
\end{array}\right], & M A(i) N & =\left[\begin{array}{ll}
A_{1}(i) & A_{2}(i) \\
A_{3}(i) & A_{4}(i)
\end{array}\right], \\
M A_{d}(i) N & =\left[\begin{array}{ll}
A_{d 1}(i) & A_{d 2}(i) \\
A_{d 3}(i) & A_{d 4}(i)
\end{array}\right], & M E_{1}(i) & =\left[\begin{array}{ll}
E_{11}(i) \\
E_{12}(i)
\end{array}\right], \quad x_{k}=N\left[\begin{array}{l}
x_{1 k} \\
x_{2 k}
\end{array}\right], \\
F_{1}(i) N & =\left[\begin{array}{lll}
F_{11}(i) & F_{12}(i)
\end{array}\right], & F_{2}(i) N & =\left[\begin{array}{ll}
F_{21}(i) & F_{22}(i)
\end{array}\right],
\end{aligned}\right.
$$


where $x_{1 k} \in R^{r}, x_{2 k} \in R^{n-r}$,

\begin{tabular}{|c|c|c|}
\hline$A_{1}(i), A_{d 1}(i) \in R^{r \times r}$ & $A_{2}(i), A_{d 2}(i) \in R^{r \times(n-r)}$ & $A_{3}(i), A_{d 3}(i) \in R^{(n-r) \times r}$ \\
\hline$A_{4}(i), A_{d 4}(i) \in R^{(n-r) \times(n-r)}$, & $E_{11}(i) \in R^{r \times q}$ & $R^{(n-r) \times q}$, \\
\hline
\end{tabular}

So System (2.1) with $u_{k}=0$ is RES to the following system for each mode $i \in \varphi$ :

$$
\left\{\begin{aligned}
x_{1(k+1)}= & \left(A_{1}(i)+E_{11}(i) \Delta(k, i) F_{11}(i)\right) x_{1 k}+\left(A_{d 1}(i)+E_{11}(i) \Delta(k, i) F_{21}(i)\right) x_{1(k-d)} \\
& +\left(A_{2}(i)+E_{11}(i) \Delta(k, i) F_{12}(i)\right) x_{2 k}+\left(A_{d 2}(i)+E_{11}(i) \Delta(k, i) F_{22}(i)\right) x_{2(k-d)}, \\
0= & \left(A_{3}(i)+E_{12}(i) \Delta(k, i) F_{11}(i)\right) x_{1 k}+\left(A_{d 3}(i)+E_{12}(i) \Delta(k, i) F_{21}(i)\right) x_{1(k-d)} \\
& +\left(A_{4}(i)+E_{12}(i) \Delta(k, i) F_{12}(i)\right) x_{2 k}+\left(A_{d 4}(i)+E_{12}(i) \Delta(k, i) F_{22}(i)\right) x_{2(k-d)} .
\end{aligned}\right.
$$

By introducing new state vectors as

$$
\hat{x}_{k}=\left[x_{1 k}^{T}, x_{2(k-1)}^{T}, x_{1(k-1)}^{T}, x_{2(k-2)}^{T}\right]^{T},
$$

then System (3.2) can be rewritten as

$$
\left(\hat{E}\left(r_{k}\right)+\delta \hat{E}\left(k, r_{k}\right)\right) \hat{x}_{k+1}=\left(\hat{A}\left(r_{k}\right)+\delta \hat{A}\left(k, r_{k}\right)\right) \hat{x}_{k}+\left(\hat{A}_{d}\left(r_{k}\right)+\delta \hat{A}_{d}\left(k, r_{k}\right)\right) \hat{x}_{k-d+1},
$$

where for $r_{k}=i, i \in \varphi$

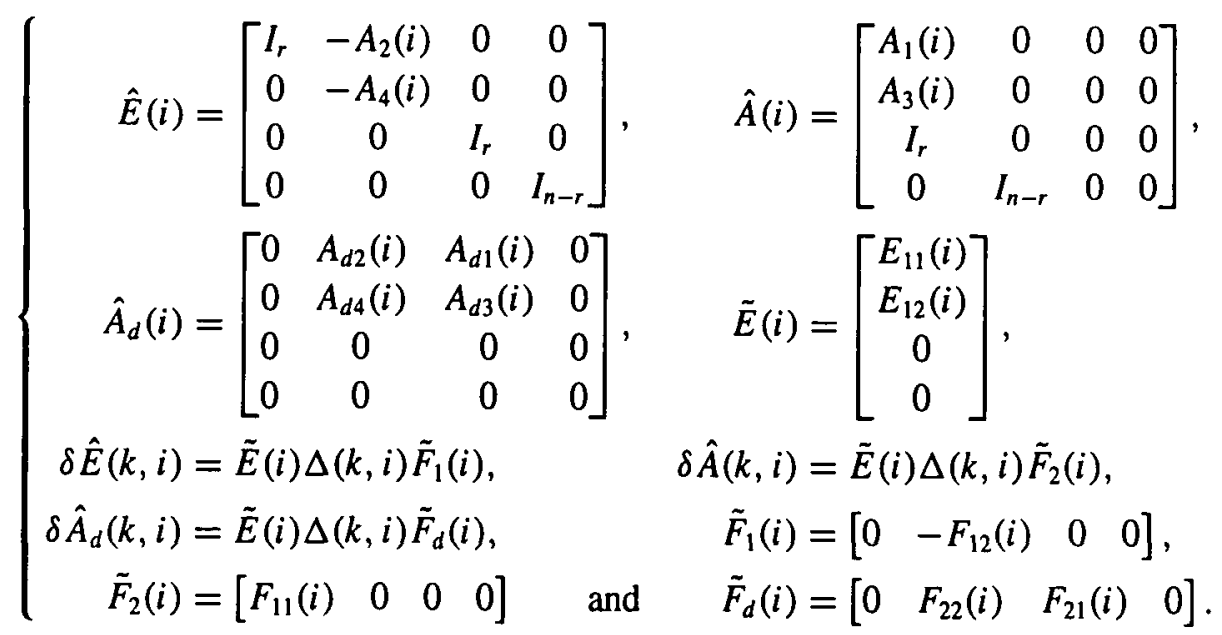

When $\Delta(k, i)=0$ in (3.4), System (2.1) with $\delta A\left(k, r_{k}\right)=0, \delta A_{d}\left(k, r_{k}\right)=0$ and $u_{k}=0$ can be written as

$$
\hat{E}\left(r_{k}\right) \hat{x}_{k+1}=\hat{A}\left(r_{k}\right) \hat{x}_{k}+\hat{A}_{d}\left(r_{k}\right) \hat{x}_{k-d+1}
$$


For the relation between System (2.1) with $\delta A\left(k, r_{k}\right)=0, \delta A_{d}\left(k, r_{k}\right)=0$ and $u_{k}=0$ and System (3.6), we have the following result.

LEMMA 3.1. System (2.1) with $\delta A\left(k, r_{k}\right)=0, \delta A_{d}\left(k, r_{k}\right)=0$ and $u_{k}=0$ is regular, causal and stochastically stable, if System (3.6) is a Markovian jump standard linear system (that is, the matrix $\hat{E}(i)$ is nonsingular for each mode $i \in \varphi$ ) and stochastically stable.

PROOF. First, consider the regularity and causality of System (2.1) which has $\delta A\left(k, r_{k}\right)=0, \delta A_{d}\left(k, r_{k}\right)=0$ and $u_{k}=0$. If System (3.6) is a Markovian jump standard linear system, from (3.5) it follows that $A_{4}(i)$ is nonsingular [9]. Then, from Definition 2.1, System (2.1) with $\delta A\left(k, r_{k}\right)=0, \delta A_{d}\left(k, r_{k}\right)=0$ and $u_{k}=0$ is regular and causal.

Next, consider the stochastic stability of System (2.1) with $\delta A\left(k, r_{k}\right)=0$, $\delta A_{d}\left(k, r_{k}\right)=0$ and $u_{k}=0$. Since System (3.6) is stochastically stable, Definition 2.2 yields

$$
\mathbf{E}\left\{\sum_{k=0}^{\infty}\left\|\hat{x}_{k}\left(\hat{\phi}, r_{0}\right)\right\|^{2} \mid \hat{\phi}, r_{0}\right\}<\infty
$$

for every initial state $\left(\hat{\phi}, r_{0}\right)$, where $\hat{\phi}(k)=\left[\phi_{1}^{T}(k) \phi_{2}^{T}(k-1) \phi_{1}^{T}(k-1) \phi_{2}^{T}(k-2)\right]^{T}$ and $\phi(k)=N\left[\begin{array}{l}\phi_{1}(k) \\ \phi_{2}(k)\end{array}\right]$. It follows that

$$
\mathrm{E}\left\{\sum_{k=0}^{\infty}\left(\left\|x_{1 k}\left(\phi_{1}, \phi_{2}, r_{0}\right)\right\|^{2}+\left\|x_{2 k}\left(\phi_{1}, \phi_{2}, r_{0}\right)\right\|^{2}\right) \mid \phi_{1}, \phi_{2}, r_{0}\right\}<\infty .
$$

Therefore, from Remark 2, System (2.1) with $\delta A\left(k, r_{k}\right)=0, \delta A_{d}\left(k, r_{k}\right)=0$ and $u_{k}=0$ is stochastically stable. The proof is completed.

REMARK 3. Based on the transformations (3.1) and (3.3), System (2.1) with $u_{k}=0$ is transformed into System (3.4) with delay $d-1$, and System (2.1) with $\delta A\left(k, r_{k}\right)=0$, $\delta A_{d}\left(k, r_{k}\right)=0$ and $u_{k}=0$ is transformed into System (3.6) with delay $d-1$. Based on Lemma 3.1, the regularity, causality and stochastic stability of System (2.1) with $\delta A\left(k, r_{k}\right)=0, \delta A_{d}\left(k, r_{k}\right)=0$ and $u_{k}=0$ and the robust stochastic stability of System (2.1) with $u_{k}=0$ can be solved by solving the stochastic stability of the standard linear system (3.6) and the robust stochastic stability of the uncertain standard linear system (3.4).

THEOREM 3.2. System (2.1) with $\delta A\left(k, r_{k}\right)=0, \delta A_{d}\left(k, r_{k}\right)=0$ and $u_{k}=0$ is regular, causal and stochastically stable, if for each mode $i \in \varphi$, there exist matrices 
$X_{i}>0, Z>0, U>0, N_{i 1}, N_{i 2}, N_{i 3}, S_{i 1}, S_{i 2}$ and $S_{i 3}$, satisfying the following $L M I$ :

$$
\begin{aligned}
\Phi_{i}=\left[\begin{array}{cccc}
\Phi_{i 11} & \Phi_{i 12} & \Phi_{i 13} & (\bar{d}-1) N_{i 1} \\
* & \Phi_{i 22} & \Phi_{i 23} & (\bar{d}-1) N_{i 2} \\
* & * & \Phi_{i 33} & (\bar{d}-1) N_{i 3} \\
* & * & * & -(\bar{d}-1) Z
\end{array}\right]<0, \text { where } \\
\Phi_{i 11}=\sum_{j=1}^{N} p_{i j} X_{j}-X_{i}+U+N_{i 1}+N_{i 1}^{T}+S_{i 1}(\hat{A}(i)-\hat{E}(i))+(\hat{A}(i)-\hat{E}(i))^{T} S_{i 1}^{T}, \\
\Phi_{i 12}=(\hat{A}(i)-\hat{E}(i))^{T} S_{i 2}^{T}-N_{i 1}+N_{i 2}^{T}+S_{i 1} \hat{A}_{d}(i), \\
\Phi_{i 13}=\sum_{j=1}^{N} p_{i j} X_{j}+N_{i 3}^{T}-S_{i 1} \hat{E}(i)+(\hat{A}(i)-\hat{E}(i))^{T} S_{i 3}^{T}, \\
\Phi_{i 22}=S_{i 2} \hat{A}_{d}(i)+\hat{A}_{d}^{T}(i) S_{i 2}^{T}-N_{i 2}-N_{i 2}^{T}-U \\
\Phi_{i 23}=\hat{A}_{d}^{T}(i) S_{i 3}^{T}-S_{i 2} \hat{E}(i)-N_{i 3}^{T} \text { and } \\
\Phi_{i 33}=\sum_{j=1}^{N} p_{i j} X_{j}+(\bar{d}-1) Z-S_{i 3} \hat{E}(i)-\hat{E}^{T}(i) S_{i 3}^{T} .
\end{aligned}
$$

PROOF. Based on Lemma 3.1, to prove that System (2.1) with $\delta A\left(k, r_{k}\right)=0$, $\delta A_{d}\left(k, r_{k}\right)=0$ and $u_{k}=0$ is regular, causal and stochastically stable, it suffices to prove that the matrix $\hat{E}(i)$ is nonsingular for each mode $i \in \varphi$ and System (3.6) is stochastically stable. First, we prove that the matrix $\hat{E}(i)$ is nonsingular for each mode $i \in \varphi$. From (3.7) it follows that $\Phi_{i 33}<0$. Since $X_{i}>0, Z>0$ and $\bar{d} \geq 1$, we obtain that $-S_{i 3} \hat{E}(i)-\hat{E}^{T}(i) S_{i 3}^{T}<0$. Then $\hat{E}(i)$ is nonsingular for each mode $i \in \varphi$.

Next, we prove that System (3.6) is stochastically stable. Rewrite System (3.6) as

$$
\begin{aligned}
\hat{x}_{k+1} & =\hat{x}_{k}+\hat{y}_{k}, \\
0 & =-\hat{E}\left(r_{k}\right) \hat{y}_{k}+\left(\hat{A}\left(r_{k}\right)-\hat{E}\left(r_{k}\right)\right) \hat{x}_{k}+\hat{A}_{d}\left(r_{k}\right) \hat{x}_{k-d+1} .
\end{aligned}
$$

Let $\hat{X}_{k}=\left(\hat{x}_{k}, \hat{x}_{k-1}, \cdots, \hat{x}_{k-d+1}\right)$ and $\hat{Y}_{k}=\left(\hat{y}_{k-1}, \cdots, \hat{y}_{k-d+1}\right)$. We may then construct a stochastic Lyapunov functional as

$$
\left\{\begin{array}{l}
V\left(\hat{X}_{k}, \hat{Y}_{k}, k, r_{k}\right)=V_{1}\left(\hat{X}_{k}, \hat{Y}_{k}, k, r_{k}\right)+V_{2}\left(\hat{X}_{k}, \hat{Y}_{k}, k, r_{k}\right)+V_{3}\left(\hat{X}_{k}, \hat{Y}_{k}, k, r_{k}\right), \\
V_{1}\left(\hat{X}_{k}, \hat{Y}_{k}, k, r_{k}\right)=\hat{x}_{k}^{T} X_{r_{k}} \hat{x}_{k}, \quad V_{2}\left(\hat{X}_{k}, \hat{Y}_{k}, k, r_{k}\right)=\sum_{l=k-d+1}^{k-1} \hat{x}_{l}^{T} U \hat{x}_{l}, \\
V_{3}\left(\hat{X}_{k}, \hat{Y}_{k}, k, r_{k}\right)=\sum_{\theta=-d+2}^{0} \sum_{l=k-1+\theta}^{k-1} \hat{y}_{l}^{T} Z \hat{y}_{l},
\end{array}\right.
$$


where the matrices $X_{r_{k}}>0, U>0$ and $Z>0$. Let the mode at time $k$ be $i$, that is, $r_{k}=i$. Recall that at time $k+1$, the system may jump to any mode $r_{k+1}=j$. One can then obtain that

$$
\begin{aligned}
& \mathbf{E}\left[V_{1}\left(\hat{X}_{k+1}, \hat{Y}_{k+1}, k+1, r_{k+1}\right) \mid \hat{X}_{k}, \hat{Y}_{k}, r_{k}=i\right]-V_{1}\left(\hat{X}_{k}, \hat{Y}_{k}, k, i\right) \\
& =\mathbf{E}\left[\hat{x}_{k+1}^{T} X_{r_{k+1}} \hat{x}_{k+1} \mid \hat{X}_{k}, \hat{Y}_{k}, r_{k}=i\right]-\hat{x}_{k}^{T} X_{i} \hat{x}_{k} \\
& =\mathrm{E}\left[\left(\hat{x}_{k}+\hat{y}_{k}\right)^{T} X_{r_{k+1}}\left(\hat{x}_{k}+\hat{y}_{k}\right) \mid \hat{X}_{k}, \hat{Y}_{k}, r_{k}=i\right]-\hat{x}_{k}^{T} X_{i} \hat{x}_{k} \\
& =\sum_{j=1}^{N} p_{i j}\left(\hat{x}_{k}+\hat{y}_{k}\right)^{T} X_{j}\left(\hat{x}_{k}+\hat{y}_{k}\right)-\hat{x}_{k}^{T} X_{i} \hat{x}_{k} \text {, } \\
& \mathbf{E}\left[V_{2}\left(\hat{X}_{k+1}, \hat{Y}_{k+1}, k+1, r_{k+1}\right) \mid \hat{X}_{k}, \hat{Y}_{k}, r_{k}=i\right]-V_{2}\left(\hat{X}_{k}, \hat{Y}_{k}, k, i\right) \\
& =\mathbf{E}\left[\sum_{l=k-d+2}^{k} \hat{x}_{l}^{T} U \hat{x}_{l} \mid \hat{X}_{k}, \hat{Y}_{k}, r_{k}=i\right]-\sum_{l=k-d+1}^{k-1} \hat{x}_{l}^{T} U \hat{x}_{l} \\
& =\hat{x}_{k}^{T} U \hat{x}_{k}-\hat{x}_{k-d+1}^{T} U \hat{x}_{k-d+1} \quad \text { and } \\
& \mathbf{E}\left[V_{3}\left(\hat{X}_{k+1}, \hat{Y}_{k+1}, k+1, r_{k+1}\right) \mid \hat{X}_{k}, \hat{Y}_{k}, r_{k}=i\right]-V_{3}\left(\hat{X}_{k}, \hat{Y}_{k}, k, i\right) \\
& =\mathrm{E}\left[\sum_{\theta=-d+2}^{0} \sum_{l=k+\theta}^{k} \hat{y}_{l}^{T} Z \hat{y}_{l} \mid \hat{X}_{k}, \hat{Y}_{k}, r_{k}=i\right]-\sum_{\theta=-d+2 l=k-1+\theta}^{0} \sum_{l=1}^{k-1} \hat{y}_{l}^{T} Z \hat{y}_{l} \\
& =(d-1) \hat{y}_{k}^{T} Z \hat{y}_{k}-\sum_{l=k-d+1}^{k-1} \hat{y}_{l}^{T} Z \hat{y}_{l} .
\end{aligned}
$$

From (3.9)-(3.11), it follows that

$$
\begin{aligned}
& \mathbf{E}\left[V\left(\hat{X}_{k+1}, \hat{Y}_{k+1}, k+1, r_{k+1}\right) \mid \hat{X}_{k}, \hat{Y}_{k}, r_{k}=i\right]-V\left(\hat{X}_{k}, \hat{Y}_{k}, k, i\right) \\
& \leq \sum_{j=1}^{N} p_{i j}\left(\hat{x}_{k}+\hat{y}_{k}\right)^{T} X_{j}\left(\hat{x}_{k}+\hat{y}_{k}\right)-\hat{x}_{k}^{T} X_{i} \hat{x}_{k}+\hat{x}_{k}^{T} U \hat{x}_{k}-\hat{x}_{k-d+1}^{T} U \hat{x}_{k-d+1} \\
& \quad+(\bar{d}-1) \hat{y}_{k}^{T} Z \hat{y}_{k}-\sum_{l=k-d+1}^{k-1} \hat{y}_{l}^{T} Z \hat{y}_{l} .
\end{aligned}
$$

From the first equation in (3.8), for any appropriate dimensions $N_{i 1}, N_{i 2}$ and $N_{i 3}$, the following equation holds:

$$
2\left(\hat{x}_{k}^{T} N_{i 1}+\hat{x}_{k-d+1}^{T} N_{i 2}+\hat{y}_{k}^{T} N_{i 3}\right)\left(\hat{x}_{k}-\hat{x}_{k-d+1}-\sum_{l=k-d+1}^{k-1} \hat{y}_{l}\right)=0 .
$$


And from the second formula of (3.8), for any appropriate dimensions $S_{i 1}, S_{i 2}$ and $S_{i 3}$, the following equation is also true for $r_{k}=i$ :

$$
2\left(\hat{x}_{k}^{T} S_{i 1}+\hat{x}_{k-d+1}^{T} S_{i 2}+\hat{y}_{k}^{T} S_{i 3}\right)\left(-\hat{E}(i) \hat{y}_{k}+(\hat{A}(i)-\hat{E}(i)) \hat{x}_{k}+\hat{A}_{d}(i) \hat{x}_{k-d+1}\right)=0 .
$$

For a semi-positive definite matrix $Q_{i}$, the following holds:

$$
(\bar{d}-1)\left[\begin{array}{c}
\hat{x}_{k} \\
\hat{x}_{k-d+1} \\
\hat{y}_{k}
\end{array}\right]^{T} Q_{i}\left[\begin{array}{c}
\hat{x}_{k} \\
\hat{x}_{k-d+1} \\
\hat{y}_{k}
\end{array}\right]-\sum_{l=k-d+1}^{k-1}\left[\begin{array}{c}
\hat{x}_{k} \\
\hat{x}_{k-d+1} \\
\hat{y}_{k}
\end{array}\right]^{T} Q_{i}\left[\begin{array}{c}
\hat{x}_{k} \\
\hat{x}_{k-d+1} \\
\hat{y}_{k}
\end{array}\right] \geq 0 .
$$

Then, adding the terms on the left of (3.13)-(3.15) to (3.12), it is obtained that

$$
\begin{aligned}
& \mathbf{E}\left[V\left(\hat{X}_{k+1}, \hat{Y}_{k+1}, k+1, r_{k+1}\right) \mid \hat{X}_{k}, \hat{Y}_{k}, r_{k}=i\right]-V\left(\hat{X}_{k}, \hat{Y}_{k}, k, i\right) \\
& \leq\left[\begin{array}{lll}
\hat{x}_{k}^{T} & \hat{x}_{k-d+1}^{T} & \hat{y}_{k}^{T}
\end{array}\right]\left(\Lambda_{i}+(\bar{d}-1) Q_{i}\right)\left[\begin{array}{lll}
\hat{x}_{k}^{T} & \hat{x}_{k-d+1}^{T} & \hat{y}_{k}^{T}
\end{array}\right]^{T} \\
& \quad-\sum_{l=k-d+1}^{k-1}\left[\begin{array}{lllll}
\hat{x}_{k}^{T} & \hat{x}_{k-d+1}^{T} & \hat{y}_{k}^{T} & \hat{y}_{l}^{T}
\end{array}\right] \Pi_{i}\left[\begin{array}{llll}
\hat{x}_{k}^{T} & \hat{x}_{k-d+1}^{T} & \hat{y}_{k}^{T} & \hat{y}_{l}^{T}
\end{array}\right]^{T},
\end{aligned}
$$

where

$$
\Lambda_{i}=\left[\begin{array}{ccc}
\Phi_{i 11} & \Phi_{i 12} & \Phi_{i 13} \\
* & \Phi_{i 22} & \Phi_{i 23} \\
* & * & \Phi_{i 33}
\end{array}\right] \text { and } \Pi_{i}=\left[\begin{array}{ccc|c} 
& & N_{i 1} \\
& Q_{i} & & N_{i 2} \\
& & & N_{i 3} \\
N_{i 1}^{T} & N_{i 2}^{T} & N_{i 3}^{T} & Z
\end{array}\right]
$$

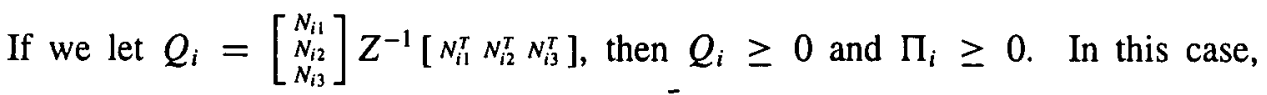
the inequality (3.7) is equivalent to $\Lambda_{i}+(\bar{d}-1) Q_{i}<0$, according to the Schur complement. Let $\alpha_{0}=\lambda_{\min }\left\{-\left(\Lambda_{i}+(\bar{d}-1) Q_{i}\right), i \in \varphi\right\}$, then $\alpha_{0}>0$. From (3.16), we obtain that for any $k \geq 0$

$$
\mathbf{E}\left[V\left(\hat{X}_{k+1}, \hat{Y}_{k+1}, k+1, r_{k+1}\right) \mid \hat{X}_{k}, \hat{Y}_{k}, r_{k}=i\right] \leq V\left(\hat{X}_{k}, \hat{Y}_{k}, k, r_{k}\right)-\alpha_{0} \hat{x}_{k}^{T} \hat{x}_{k} .
$$

Setting $k=0$ and $k=1$ in (3.17) yields

and

$$
\mathbf{E}\left[V\left(\hat{X}_{1}, \hat{Y}_{1}, 1, r_{1}\right) \mid \hat{X}_{0}, \hat{Y}_{0}, r_{0}\right] \leq V\left(\hat{X}_{0}, \hat{Y}_{0}, 0, r_{0}\right)-\alpha_{0} \hat{x}_{0}^{T} \hat{x}_{0}
$$

$$
\mathbf{E}\left[V\left(\hat{X}_{2}, \hat{Y}_{2}, 2, r_{2}\right) \mid \hat{X}_{1}, \hat{Y}_{1}, r_{1}\right] \leq V\left(\hat{X}_{1}, \hat{Y}_{1}, 1, r_{1}\right)-\alpha_{0} \hat{x}_{1}^{T} \hat{x}_{1}
$$


[11] Stability \& stabilization of uncertain time-delay discrete Markovian jump singular systems

Taking the expectation $\mathbf{E}\left[\cdot \mid \hat{X}_{0}, \hat{Y}_{0}, r_{0}\right]$ on both sides of (3.18) with the aid of (3.19), leads to

$$
\mathbf{E}\left[V\left(\hat{X}_{2}, \hat{Y}_{2}, 2, r_{2}\right) \mid \hat{X}_{0}, \hat{Y}_{0}, r_{0}\right] \leq V\left(\hat{X}_{0}, \hat{Y}_{0}, 0, r_{0}\right)-\alpha_{0} \sum_{l=0}^{1} \mathbf{E}\left[\hat{x}_{l}^{T} \hat{x}_{l} \mid \hat{X}_{0}, \hat{Y}_{0}, r_{0}\right]
$$

Then, one can continue the iterative procedure (3.17) to obtain

$$
\begin{aligned}
& \mathbf{E}\left[V\left(\hat{X}_{T+1}, \hat{Y}_{T+1}, T+1, r_{T+1}\right) \mid \hat{X}_{0}, \hat{Y}_{0}, r_{0}\right] \\
& \quad \leq V\left(\hat{X}_{0}, \hat{Y}_{0}, 0, r_{0}\right)-\alpha_{0} \sum_{l=0}^{T} \mathbf{E}\left[\hat{x}_{l}^{T} \hat{x}_{l} \mid \hat{X}_{0}, \hat{Y}_{0}, r_{0}\right]
\end{aligned}
$$

implying that

$$
\sum_{l=0}^{\infty} \mathbf{E}\left[\hat{x}_{l}^{T} \hat{x}_{l} \mid \hat{X}_{0}, \hat{Y}_{0}, r_{0}\right] \leq \frac{1}{\alpha_{0}} V\left(\hat{X}_{0}, \hat{Y}_{0}, 0, r_{0}\right)<\infty
$$

This indicates that System (3.6) is stochastically stable. The proof is completed.

Considering the regularity, causality and robust stochastic stability of System (2.1) with $u_{k}=0$ for all uncertainties satisfying (2.2) and (2.3), the following theorem is given.

THEOREM 3.3. System (2.1) with $u_{k}=0$ is regular, causal and stochastically stable for all uncertainties satisfying (2.2) and (2.3), if for each mode $i \in \varphi$, there exist matrices $X_{i}>0, Z>0, U>0, N_{i 1}, N_{i 2}, N_{i 3}, S_{i 1}, S_{i 2}$ and $S_{i 3}$, and scalars $\lambda_{i}>0$ satisfying the following $L M I$ :

$$
\left[\begin{array}{ccc}
\Phi_{i} & \Pi_{i a} & \lambda_{i} \Pi_{i b}^{T} \\
* & -\lambda_{i} I & 0 \\
* & * & -\lambda_{i} I
\end{array}\right]<0
$$

where

$$
\begin{aligned}
& \Pi_{i a}=\left[\begin{array}{llll}
\left(S_{i 1} \tilde{E}(i)\right)^{T} & \left(S_{i 2} \tilde{E}(i)\right)^{T} & \left(S_{i 3} \tilde{E}(i)\right)^{T} & 0
\end{array}\right]^{T} \text { and } \\
& \Pi_{i b}=\left[\begin{array}{llll}
\tilde{F}_{2}(i)-\tilde{F}_{1}(i) & \tilde{F}_{d}(i) & -\tilde{F}_{1}(i) & 0
\end{array}\right] .
\end{aligned}
$$

PROOF. From Theorem 3.2, replacing $\hat{E}(i), \hat{A}(i)$ and $\hat{A}_{d}(i)$ in (3.7) with $\hat{E}(i)+\delta \hat{E}(k, i), \hat{A}(i)+\delta \hat{A}(k, i)$ and $\hat{A}_{d}(i)+\delta \hat{A}_{d}(k, i)$, respectively, then System (2.1) with $u_{k}=0$ is regular, causal and stochastically stable for all uncertainties satisfying (2.2) and (2.3). According to (3.5), the inequality (3.7) can be written as

$$
\Phi_{i}+\Pi_{i a} \Delta(k, i) \Pi_{i b}+\left(\Pi_{i a} \Delta(k, i) \Pi_{i b}\right)^{T}<0 .
$$


By Lemma 2.3, a sufficient condition for (3.21) is that there exists a scalar $\lambda_{i}>0$ such that

$$
\Phi_{i}+\lambda_{i}^{-1} \Pi_{i a} \Pi_{i a}^{T}+\lambda_{i} \Pi_{i b}^{T} \Pi_{i b}<0
$$

Applying the Schur complement shows that (3.22) is equivalent to (3.20). The proof is completed.

In the following, we design a robust state feedback controller in the form of (2.4) for System (2.1) such that the resulting closed-loop system is regular, causal and robust stochastically stable for all uncertainties satisfying (2.2) and (2.3). The closed-loop system formed by System (2.1) and the state feedback control law $u_{k}=K\left(r_{k}\right) x_{k}$ is

$$
E x_{k+1}=\left(A\left(k, r_{k}\right)+B\left(k, r_{k}\right) K\left(r_{k}\right)\right) x_{k}+A_{d}\left(k, r_{k}\right) x_{k-d} .
$$

By the transformation (3.1), for $r_{k}=i, i \in \varphi$, the closed-loop system (3.23) is RES to the following system:

$$
\left\{\begin{aligned}
x_{1(k+1)}= & \left(A_{1 K}(i)+E_{11}(i) \Delta(k, i) F_{1 K}(i)\right) x_{1 k}+\left(A_{2 K}(i)+E_{11}(i) \Delta(k, i) F_{2 K}(i)\right) x_{2 k} \\
& +\left(A_{d 1}(i)+E_{11}(i) \Delta(k, i) F_{21}(i)\right) x_{1(k-d)} \\
& +\left(A_{d 2}(i)+E_{11}(i) \Delta(k, i) F_{22}(i)\right) x_{2(k-d)} \\
0= & \left(A_{3 K}(i)+E_{12}(i) \Delta(k, i) F_{1 K}(i)\right) x_{1 k}+\left(A_{4 K}(i)+E_{12}(i) \Delta(k, i) F_{2 K}(i)\right) x_{2 k} \\
& +\left(A_{d 3}(i)+E_{12}(i) \Delta(k, i) F_{21}(i)\right) x_{1(k-d)} \\
& +\left(A_{d 4}(i)+E_{12}(i) \Delta(k, i) F_{22}(i)\right) x_{2(k-d)}
\end{aligned}\right.
$$

where

$$
\begin{array}{ll}
A_{1 K}(i)=A_{1}(i)+B_{1}(i) K_{1}(i), & A_{2 K}(i)=A_{2}(i)+B_{1}(i) K_{2}(i), \\
A_{3 K}(i)=A_{3}(i)+B_{2}(i) K_{1}(i), & A_{4 K}(i)=A_{4}(i)+B_{2}(i) K_{2}(i), \\
F_{1 K}(i)=F_{11}(i)+F_{3}(i) K_{1}(i), & F_{2 K}(i)=F_{12}(i)+F_{3}(i) K_{2}(i), \\
M B(i)=\left[\begin{array}{ll}
B_{1}(i) \\
B_{2}(i)
\end{array}\right], & K(i) N=\left[\begin{array}{ll}
K_{1}(i) & K_{2}(i)
\end{array}\right],
\end{array}
$$

and $B_{1}(i) \in R^{r \times p}, B_{2}(i) \in R^{(n-r) \times p}, K_{1}(i) \in R^{p \times r}$ and $K_{2}(i) \in R^{p \times(n-r)}$. From (3.3), System (3.24) is rewritten as

$$
\left(\hat{E}_{K}\left(r_{k}\right)+\delta \hat{E}_{K}\left(k, r_{k}\right)\right) \hat{x}_{k+1}=\left(\hat{A}_{K}\left(r_{k}\right)+\delta \hat{A}_{K}\left(k, r_{k}\right)\right) \hat{x}_{k}+\left(\hat{A}_{d}\left(r_{k}\right)+\delta \hat{A}_{d}\left(k, r_{k}\right)\right) \hat{x}_{k-d+1},
$$



where, for any $r_{k}=i, i \in \varphi$,

$$
\begin{aligned}
\hat{E}_{K}(i) & =\hat{E}(i)-\hat{B}(i) \hat{K}_{2}(i), & \hat{A}_{K}(i) & =\hat{A}(i)+\hat{B}(i) \hat{K}_{1}(i), \\
\delta \hat{E}_{K}(k, i) & =\tilde{E}(i) \Delta(k, i) \tilde{F}_{1 K}(i), & \delta \hat{A}_{K}(k, i) & =\tilde{E}(i) \Delta(k, i) \tilde{F}_{2 K}(i), \\
\tilde{F}_{1 K}(i) & =\tilde{F}_{1}(i)-F_{3}(i) \hat{K}_{2}(i), & \tilde{F}_{2 K}(i) & =\tilde{F}_{2}(i)+F_{3}(i) \hat{K}_{1}(i), \\
\hat{B}(i) & =\left[\begin{array}{llllll}
B_{1}^{T}(i) & B_{2}^{T}(i) & 0 & 0
\end{array}\right]^{T}, & \hat{K}_{1}(i) & =\left[\begin{array}{lllll}
K_{1}(i) & 0 & 0 & 0
\end{array}\right], \\
\hat{K}_{2}(i) & =\left[\begin{array}{lllll}
0 & K_{2}(i) & 0 & 0
\end{array}\right], & &
\end{aligned}
$$

and $\hat{E}(i), \hat{A}(i), \tilde{F}_{1}(i), \tilde{F}_{2}(i), \tilde{E}(i), \hat{A}_{d}(i)$ and $\delta \hat{A}_{d}(k, i)$ are shown as in (3.5).

THEOREM 3.4. If for each $i \in \varphi$, and given scalars $t_{i 1}, t_{i 2}, t_{i 3}$, there exist matrices $\bar{X}_{i}>0, \bar{Z}>0, \bar{U}>0, \bar{N}_{i 1}, \bar{N}_{i 2}, \bar{N}_{i 3}, \bar{K}_{1}(i), \bar{K}_{2}(i)$ and $R=\operatorname{diag}\left\{R_{11}, R_{22}, R_{2}\right\}$, $R_{11} \in R^{r \times r}, R_{22} \in R^{(n-r) \times(n-r)}$ is nonsingular, $R_{2} \in R^{n \times n}$, and scalar $\epsilon_{i}>0$ satisfying the following LMI:

$\left[\begin{array}{cccccc}\Psi_{i 11} & \Psi_{i 12} & \Psi_{i 13} & (\bar{d}-1) \bar{N}_{i 1} & \epsilon_{i} t_{i 1} \tilde{E}(i) & \left(\Psi_{f 2}(i)-\Psi_{f 1}(i)\right)^{T} \\ * & \Psi_{i 22} & \Psi_{i 23} & (\bar{d}-1) \bar{N}_{i 2} & \epsilon_{i} t_{i 2} \tilde{E}(i) & \left(\tilde{F}_{d}(i) R\right)^{T} \\ * & * & \Psi_{i 33} & (\bar{d}-1) \bar{N}_{i 3} & \epsilon_{i} t_{i 3} \tilde{E}(i) & -\Psi_{f 1}^{T}(i) \\ * & * & * & -(\bar{d}-1) \bar{Z} & 0 & 0 \\ * & * & * & * & -\epsilon_{i} I & 0 \\ * & * & * & * & * & -\epsilon_{i} I\end{array}\right]<0$,

where

$$
\begin{aligned}
& \Psi_{i 11}=\sum_{j=1}^{N} p_{i j} \bar{X}_{j}-\bar{X}_{i}+\bar{U}+\bar{N}_{i 1}+\bar{N}_{i 1}^{T}+t_{i 1}\left(\Psi_{a}(i)-\Psi_{e}(i)\right)+t_{i 1}\left(\Psi_{a}(i)-\Psi_{e}(i)\right)^{T}, \\
& \Psi_{i 12}=t_{i 2}\left(\Psi_{a}(i)-\Psi_{e}(i)\right)^{T}-\bar{N}_{i 1}+\bar{N}_{i 2}^{T}+t_{i 1} \hat{A}_{d}(i) R, \\
& \Psi_{i 13}=\sum_{j=1}^{N} p_{i j} \bar{X}_{j}+\bar{N}_{i 3}^{T}-t_{i 1} \Psi_{e}(i)+t_{i 3}\left(\Psi_{a}(i)-\Psi_{e}(i)\right)^{T}, \\
& \Psi_{i 22}=t_{i 2} \hat{A}_{d}(i) R+t_{i 2} R^{T} \hat{A}_{d}^{T}(i)-\bar{N}_{i 2}-\bar{N}_{i 2}^{T}-\bar{U}, \\
& \Psi_{i 23}=t_{i 3} R^{T} \hat{A}_{d}^{T}(i)-t_{i 2} \Psi_{e}(i)-\bar{N}_{i 3}^{T}, \\
& \Psi_{i 33}=\sum_{j=1}^{N} p_{i j} \bar{X}_{j}+(\bar{d}-1) \bar{Z}-t_{i 3} \Psi_{e}(i)-t_{i 3} \Psi_{e}^{T}(i), \\
& \Psi_{e}(i)=\hat{E}(i) R-\hat{B}(i)\left[\begin{array}{llll}
0 & \bar{K}_{2}(i) & 0 & 0
\end{array}\right], \\
& \Psi_{a}(i)=\hat{A}(i) R+\hat{B}(i)\left[\bar{K}_{1}(i) \quad 0 \quad 0 \quad 0\right] \text {, } \\
& \Psi_{f 1}(i)=\tilde{F}_{1}(i) R-F_{3}(i)\left[\begin{array}{llll}
0 & \bar{K}_{2}(i) & 0 & 0
\end{array}\right] \text { and } \\
& \Psi_{f 2}(i)=\tilde{F}_{2}(i) R+F_{3}(i)\left[\bar{K}_{1}(i) \quad 0 \quad 0 \quad 0\right] \text {, }
\end{aligned}
$$


then there exists a robust state feedback controller for System (2.1) such that the resulting closed-loop system is regular, causal and robust stochastically stable for all uncertainties satisfying (2.2) and (2.3), and $K(i)=\left[\begin{array}{ll}\bar{K}_{1}(i) R_{11}^{-1} & \bar{K}_{2}(i) R_{22}^{-1}\end{array}\right] N^{-1}$.

PRoof. By Theorem 3.3, replacing $\hat{E}(i), \hat{A}(i), \tilde{F}_{1}(i)$ and $\tilde{F}_{2}(i)$ in (3.20) with $\hat{E}_{K}(i), \hat{A}_{K}(i), \tilde{F}_{1 K}(i)$ and $\tilde{F}_{2 K}(i)$, respectively, then the closed-loop system (3.23) is regular, causal and robust stochastically stable for all uncertainties satisfying (2.2) and (2.3). Setting $S_{i 1}^{T}=t_{i 1} S, S_{i 2}^{T}=t_{i 2} S$ and $S_{i 3}^{T}=t_{i 3} S$, then from LMI (3.20) it follows that

$$
\sum_{j=1}^{N} p_{i j} X_{j}+(\bar{d}-1) Z-t_{i 3}\left(\hat{E}_{K}^{T}(i) S+S^{T} \hat{E}_{K}(i)\right)<0 .
$$

Since $X_{i}>0, Z>0$ and $\bar{d} \geq 1$, the same is true for $-t_{i 3}\left(\hat{E}_{K}^{T}(i) S+S^{T} \hat{E}_{K}(i)\right)$. So the matrix $S$ is nonsingular. We set

$$
T=\operatorname{diag}\left\{S^{-T}, S^{-T}, S^{-T}, S^{-T}, \lambda_{i}^{-1} I, \lambda_{i}^{-1} I\right\} .
$$

Pre- and post-multipling the inequality (3.20) by $T$ and $T^{T}$, respectively, and setting

$$
\begin{aligned}
& S^{-T} U S^{-1}=\bar{U}, \quad S^{-T} Z S^{-1}=\bar{Z}, \quad S^{-T} X_{i} S^{-1}=\bar{X}_{i}, \quad S^{-T} N_{i 1} S^{-1}=\bar{N}_{i 1}, \\
& S^{-T} N_{i 2} S^{-1}=\bar{N}_{i 2}, \quad S^{-T} N_{i 3} S^{-1}=\bar{N}_{i 3}, \quad S^{-1}=R \quad \text { and } \quad \lambda_{i}^{-1}=\epsilon_{i} \text {, }
\end{aligned}
$$

and taking $R=\operatorname{diag}\left\{R_{11}, R_{22}, R_{2}\right\}, R_{11} \in R^{r \times r}, R_{22} \in R^{(n-r) \times(n-r)}, R_{2} \in R^{n \times n}$, and setting $\bar{K}_{1}(i)=K_{1}(i) R_{11}$ and $\bar{K}_{2}(i)=K_{2}(i) R_{22}$, then LMI (3.25) is obtained.

Obviously, from (3.26), the matrix $R$ in (3.25) should be nonsingular. The nonsingularity of $R$ can be obtained by the following discussion. Since $\Psi_{i 33}<0$ in (3.25), the same is true for $-t_{i 3}\left(\Psi_{e}(i)+\Psi_{e}^{T}(i)\right)$. So $R_{11}$ and $R_{2}$ are nonsingular, and $K_{1}(i)=\bar{K}_{1}(i) R_{11}^{-1}$. Since $R_{22}$ is nonsingular, $K_{2}(i)=\bar{K}_{2}(i) R_{22}^{-1}$. Thus $R$ is nonsingular, and from (3.1), it follows that $K(i)=\left[\bar{K}_{1}(i) R_{11}^{-1} \quad \bar{K}_{2}(i) R_{22}^{-1}\right] N^{-1}$. The proof is completed.

REMARK 4. The conditions (3.7), (3.20) and (3.25) given in Theorems 3.2, 3.3 and 3.4, respectively are LMIs and delay dependent. From the proof of Theorem 3.2, it is known that if the LMIs (3.7), (3.20) and (3.25) hold for $\bar{d}$, then they hold for any $d \in(0, \bar{d}]$. Generally, in delay-dependent LMI conditions, $\bar{d}$ is not very large, and the size of $\bar{d}$ can be obtained by using a numerical optimization algorithm. If the delay $d$ is known, then $\bar{d}$ in LMIs (3.7), (3.20) and (3.25) can be changed to $d$, directly.

REMARK 5. The optimal values of the tuning parameters $t_{i 1}, t_{i 2}$ and $t_{i 3}$, that were introduced in Theorem 3.4, can be found by the approach stated in [11, Remark 5]. A numerical solution to this problem can be obtained by using a numerical optimization algorithm, such as fminsearch in the Optimization Toolbox version 2.2 of Matlab 6.5. 


\section{Example}

Consider the following uncertain time-delay discrete-time singular system:

$$
\begin{aligned}
E & =\left[\begin{array}{ll}
1 & 0 \\
0 & 0
\end{array}\right], \quad A(1)=\left[\begin{array}{ll}
2 & 1 \\
2 & 3
\end{array}\right], \quad A_{d}(1)=\left[\begin{array}{cc}
0.1 & -0.5 \\
0.3 & 0.1
\end{array}\right], \quad B(1)=\left[\begin{array}{l}
-2 \\
-5
\end{array}\right], \\
E_{1}(1) & =\left[\begin{array}{l}
0.02 \\
0.01
\end{array}\right], \quad F_{1}(1)=\left[\begin{array}{ll}
0.01 & 0.01
\end{array}\right], \quad F_{2}(1)=\left[\begin{array}{ll}
0.01 & 0.02
\end{array}\right], \quad F_{3}(1)=0.01, \\
A(2) & =\left[\begin{array}{cc}
0 & 2 \\
-2 & -2
\end{array}\right], \quad A_{d}(2)=\left[\begin{array}{cc}
0.1 & -0.1 \\
0 & -0.1
\end{array}\right], \quad B(2)=\left[\begin{array}{l}
0 \\
1
\end{array}\right], \\
E_{1}(2) & =\left[\begin{array}{c}
0 \\
-0.02
\end{array}\right], \quad F_{1}(2)=\left[\begin{array}{ll}
0.005 & 0.01
\end{array}\right], \quad F_{2}(2)=\left[\begin{array}{ll}
0 & -0.01
\end{array}\right] \quad \text { and } \\
F_{3}(2) & =0.01 .
\end{aligned}
$$

Let $p_{11}=0.6, p_{12}=0.4, p_{21}=0.1$ and $p_{22}=0.9$. Also let $\bar{d}=2, t_{11}=0.2$, $t_{12}=-0.005, t_{13}=4, t_{21}=0.08, t_{22}=-0.002$ and $t_{23}=4$. Solving the LMI (3.25), we find that the LMI is feasible with the following results:

$$
\begin{aligned}
\bar{X}(1) & =10^{3} \times\left[\begin{array}{cccc}
0.3106 & 0.7862 & -0.3578 & -0.0665 \\
0.7862 & 2.5743 & -0.1292 & 0.1660 \\
-0.3578 & -0.1292 & 2.6592 & 0.5720 \\
-0.0665 & 0.1660 & 0.5720 & 2.9200
\end{array}\right], \\
\bar{X}(2) & =10^{3} \times\left[\begin{array}{cccc}
0.3168 & 0.8177 & -0.2841 & -0.0292 \\
0.8177 & 2.7065 & 0.0255 & 0.2522 \\
-0.2841 & 0.0255 & 2.3174 & 0.3934 \\
-0.0292 & 0.2522 & 0.3934 & 2.9071
\end{array}\right], \\
\bar{Z} & =10^{3} \times\left[\begin{array}{cccc}
0.1417 & 0.1335 & 0.2628 & 0.0131 \\
0.1335 & 1.0908 & -0.0967 & -0.1284 \\
0.2628 & -0.0967 & 2.0468 & 0.3167 \\
0.0131 & -0.1284 & 0.3167 & 1.2122
\end{array}\right], \\
\bar{U} & =\left[\begin{array}{cccc}
30.8316 & 79.8609 & 1.9475 & -2.1030 \\
79.8609 & 256.1200 & -13.4121 & -1.6061 \\
1.9475 & -13.4121 & 51.5632 & -4.6103 \\
-2.1030 & -1.6061 & -4.6103 & 167.8996
\end{array}\right],
\end{aligned}
$$




$$
\begin{aligned}
& \bar{N}_{11}=10^{3} \times\left[\begin{array}{cccc}
-0.0855 & 0.0974 & -0.5162 & -0.0899 \\
-0.1381 & -0.7574 & -0.3033 & -0.0464 \\
0.2079 & 0.7889 & -1.2318 & -0.2018 \\
0.0643 & 0.1667 & -0.1063 & -0.2469
\end{array}\right], \\
& \bar{N}_{12}=10^{3} \times\left[\begin{array}{cccc}
0.0796 & -0.1110 & 0.6021 & 0.1035 \\
0.1314 & 0.7398 & 0.5585 & 0.0870 \\
-0.1788 & -0.8147 & 1.3272 & 0.2512 \\
-0.0510 & -0.1611 & 0.1448 & 0.2321
\end{array}\right],
\end{aligned}
$$

$$
\bar{N}_{13}=\left[\begin{array}{cccc}
63.1533 & 96.7806 & 233.2663 & 31.0404 \\
82.3140 & 234.4307 & 335.9226 & -38.4231 \\
-0.2822 & 40.9476 & 11.0330 & -25.2583 \\
1.3931 & 139.5422 & 45.4164 & 70.2487
\end{array}\right]
$$$$
\bar{N}_{21}=\left[\begin{array}{cccc}
-82.8138 & -42.1154 & -24.5453 & 4.8740 \\
-33.5148 & -300.7799 & 14.5256 & 11.7652 \\
-24.7109 & 12.8396 & -254.0699 & -4.1169 \\
3.3901 & 9.9484 & -2.9763 & -271.5547
\end{array}\right] \text {, }
$$

$$
\bar{N}_{22}=\left[\begin{array}{cccc}
73.3869 & -6.4080 & 23.9660 & -0.1047 \\
7.7969 & 145.9444 & 1.3366 & 1.3191 \\
23.8032 & 3.1923 & 236.1654 & 7.1016 \\
-0.6818 & 0.2924 & 4.3697 & 185.9904
\end{array}\right]
$$

$$
\bar{N}_{23}=\left[\begin{array}{cccc}
31.6346 & 39.6644 & 227.6621 & 35.7815 \\
1.3799 & 23.9879 & -9.0751 & -95.7348 \\
2.2760 & -6.3680 & 46.0983 & 7.4269 \\
17.0508 & 74.9889 & 55.9022 & 43.6824
\end{array}\right]
$$

$$
R=\left[\begin{array}{cccc}
79.5754 & 0 & 0 & 0 \\
0 & -110.9884 & 0 & 0 \\
0 & 0 & 602.1076 & 103.4690 \\
0 & 0 & 131.4476 & 739.8340
\end{array}\right]
$$

$$
\begin{array}{rlrl}
\epsilon_{1} & =918.5972, & \epsilon_{2} & =1.1066 \times 10^{3}, \\
\bar{K}_{2}(1) & =70.3455, \quad \bar{K}_{1}(1)=76.1471 \\
\bar{K}_{1}(2) & =-36.2810 \text { and } & \bar{K}_{2}(2) & =-885.4138
\end{array}
$$


Then $K_{1}(1)=0.9569, K_{2}(1)=-0.6338, K_{1}(2)=-0.4559$ and $K_{2}(2)=7.9775$. Thus

$$
K(1)=\left[\begin{array}{ll}
0.9569 & -0.6338
\end{array}\right] \text { and } K(2)=\left[\begin{array}{ll}
-0.4559 & 7.9775
\end{array}\right]
$$

Figure 1 gives the simulation result of the closed-loop system. The initial function

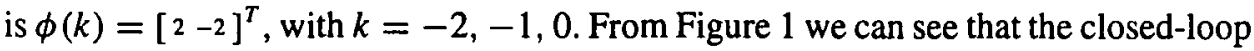
system is stable.

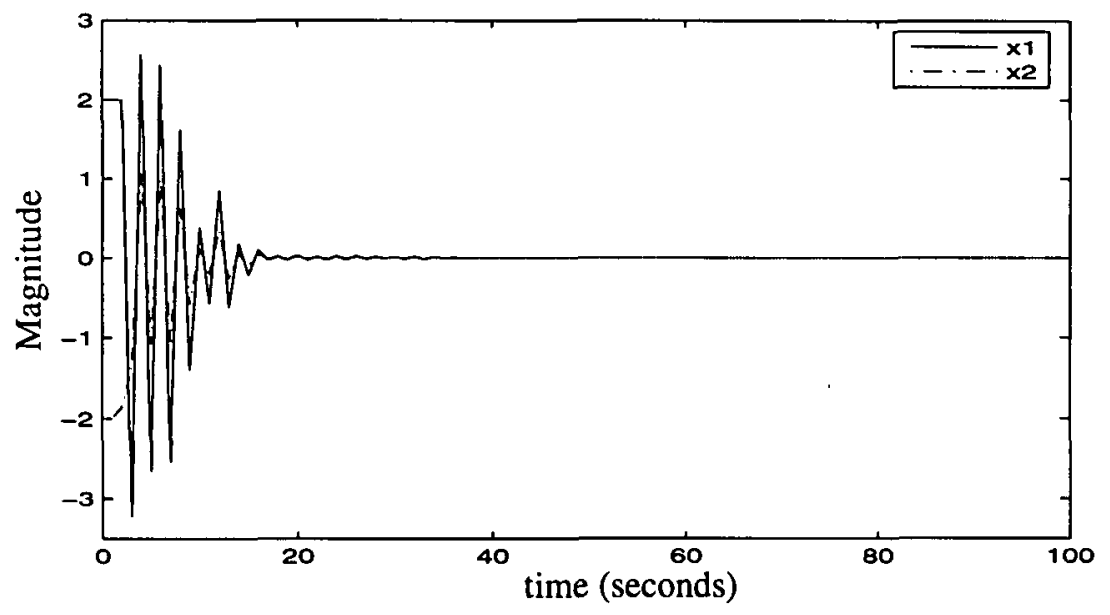

FIGURE 1. The state trajectories of the closed-loop system.

\section{Conclusions}

In this paper, we have investigated robust stochastic stability and stabilization via state feedback for time-delay discrete-time Markovian jump singular systems with parameter uncertainties. Based on the RES transformation and by introducing new state vectors, we have transformed the singular system into a time-delay discrete Markovian jump standard linear system, and then established some delay-dependent LMI conditions for time-delay discrete-time Markovian jump singular systems to be regular, causal and stochastically stable. This condition has solved the problems of robust stochastic stability and stabilization in terms of delay-dependent LMIs. We have also illustrated the effectiveness of our results by a numerical example. The results obtained in this paper may be extended and generalized to a large class of quasilinear and nonlinear problems. 
In this paper, we have only discussed the case of the singular matrix $E$ having no jump mode. If $E$ also has a jump mode, then $E$ is changed to $E_{i}$. In this case, the transformation matrices in (3.1) become $M_{i}$ and $N_{i}$, and we have

$$
M_{i} E_{i} N_{i}=\left[\begin{array}{cc}
I_{i} & 0 \\
0 & 0
\end{array}\right] \text { and } x_{k}=N_{i}\left[\begin{array}{c}
x_{1 k i} \\
x_{2 k i}
\end{array}\right]
$$

and the state of the transformed system becomes $\left[\begin{array}{l}x_{1 k t} \\ x_{2 k i}\end{array}\right]$, which is rather complicated to discuss. Hence, some assumptions for the matrix $E_{i}$ should be given so that the matrix $N_{i}$ remains the same, and the method given in this paper is invalid. Nonetheless, the case with $E$ having a jump mode is an interesting problem for future investigation via other methods.

\section{Acknowledgements}

The authors wish to thank the anonymous reviewers whose valuable comments and suggestions have improved the quality of this paper.

This work was supported by the National Science Foundation of China (10671112), the Postdoctoral Science Foundation of China (20060400980) and Shandong Province (200603015).

This research was carried out while XL was visiting Shandong University.

\section{References}

[1] J. D. Aplevich, Implicit Linear Systems (Springer-Verlag, Berlin, 1991).

[2] E. K. Boukas, "Static output feedback control for stochastic hybrid systems: LMI approach", Automatica J. IPAC 42 (2006) 183-188.

[3] E. K. Boukas and N. F. Al-Muthairi, "Delay-dependent stabilization of singular linear systems with delays", Int. J. Innovative Comput. Information Contr. 2 (2006) 283-291.

[4] E. K. Boukas and Z. K. Liu, "Robust stability and $H_{\infty}$ control of discrete-time jump linear systems with time-delays: an LMI approach", in Proceedings of the 39th IEEE Conference on Decision and Control, Sydney, Australia, 1999), 1527-1532.

[5] E. K. Boukas and Z. K. Liu, "Robust $H_{\infty}$ control of discrete-time Markovian jump linear systems with mode-dependent time-delays", IEEE Trans. Automat. Contr. 46 (2001) 1918-1924.

[6] Y. Y. Cao and J. Lams, "Stochastic stabilizability and $H_{\infty}$ control for discrete-time jump linear systems with time delay", J. Franklin Inst. 336 (1999) 1263-1281.

[7] B. Chen, J. Lam and S. Xu, "Memory state feedback guaranteed cost control for neutral delay systems", Int. J. Innovative Comput. Information Contr. 2 (2006) 293-303.

[8] W. H. Chen, Z. H. Guan and P. Yu, "Delay-dependent stability and $H_{\infty}$ control of uncertain discrete-time Markovian jump systems with mode-dependent time delays", Systems Control Lett. 52 (2004) 361-376. 
[9] L. Dai, Singular Control Systems. Lecture Notes in Control and Information Sciences (SpringerVerlag, New York, 1989).

[10] E. Fridman and U. Shaked, "A descriptor system approach to $H_{\infty}$ control of linear time-delay systems", IEEE Trans. Automat. Contr. 47 (2002) 253-270.

[11] E. Fridman and U. Shaked, "An improved stabilization method for linear time-delay systems", IEEE Trans. Automat. Contr. 47 (2002) 1931-1937.

[12] Y. He, M. Wu, J. H. She and G. P. Liu, "Delay-dependent robust stability criteria for uncertain neutral systems with mixed delays", Systems Control Lett. 51 (2004) 57-65.

[13] Y. He, M. Wu, J. H. She and G. P. Liu, "Parameter-dependent Lyapunov functional for stability of time-delay systems with polytopic type uncertainties", IEEE Trans. Automat. Contr. 49 (2004) 828-832.

[14] S. P. Ma and Z. L. Cheng, "An LMI approach to robust stabilization for uncertain discrete-time singular systems", in Proceedings of the 4Ist IEEE CDC, Las Vegas, Nevada, USA, 2002), 10901095.

[15] S. P. Ma and Z. L. Cheng, "Delay-dependent robust stabilization for uncertain discrete-time singular systems with time-delay", in Proceedings of the Sixth World Congress on Intelligent Control and Automation, Dalian, China, 2006), 2081-2085.

[16] I. R. Petersen, "A stabilization algorithm for a class of uncertain linear systems", Systems Control Lett. 8 (1987) 351-357.

[17] P. Shi and E. K. Boukas, "On $H_{\infty}$ control design for singular continuous-time delay systems with parametric uncertainties", Nonlinear Dyn. Syst. Theory 4 (2004) 59-71.

[18] P. Shi, E. K. Boukas and K. Agarwal, "Control of Markovian jump discrete-time systems with norm bounded uncertainty and unknown delay", IEEE Trans. Automat. Contr. 44 (1999) 2139-2144.

[19] M. Wu, Y. He and J. H. She, "New delay-dependent stability criteria and stabilizing method for neutral systems", IEEE Trans. Automat. Contr. 49 (2004) 2266-2271.

[20] M. Wu, Y. He, J. H. She and G. P. Liu, "Delay-dependent criteria for robust stability of time-varying delay systems", Automatica J. IPAC $\mathbf{4 0}$ (2004) 1435-1439.

[21] S. Xu, P. V. Dooren, R. Stefan and J. Lam, "Robust stability and stabilization for singular systems with state delay and parameter uncertainty", IEEE Trans. Automat. Contr. 47 (2002) 1122-1128.

[22] S. Xu and J. Lam, "Robust stability and stabilization of discrete singular systems: An equivalent characterization", IEEE Trans. Automat. Contr. 49 (2004) 568-574.

[23] S. Xu, J. Lam and C. Yang, "Robust $H_{\infty}$ control for discrete singular systems with state delay and parameter uncertainty", Dyn. Contin. Discrete Impuls. Syst Ser. B Appl. Algorithms 9 (2002) 539-554.

[24] D. Yue, J. Lam and D. W. C. Ho, "Reliable $H_{\infty}$ control of uncertain descriptor systems with multiple delays", IEE Proceedings - Control Theory and Applications 150 (2003) 557-564. 